\title{
Deep level transient spectroscopy (DLTS) of a poly(p-phenylene vinylene) Schottky diode
}

\author{
A.J. Campbell ${ }^{\text {a, }}{ }^{\text {, D.D.C. Bradley }}{ }^{\mathrm{a}}$, E. Werner ${ }^{\mathrm{b}, 1}$, W. Brütting ${ }^{\mathrm{b}}$ \\ ${ }^{a}$ Department of Physics and Astronomy, University of Sheffield, Hicks Building, Hounsfield Road, Sheffield, S3 7RH, UK \\ ${ }^{\mathrm{b}}$ Lehrstuhl für Experimentalphysik II, Universität Bayreuth, 95440 Bayreuth, Germany
}

\begin{abstract}
Deep level transient spectroscopy measurements have been carried out on ITO/poly( $p$-phenylenevinylene)/Al organic light emitting diodes that have a depletion region type Schottky barrier at the polymer/metal interface. The very long lived capacitance transients can be successfully described by the de-trapping of p-type majority carriers from a single energy trap level to a Gaussian distribution of transport states. The Gaussian width of $0.10 \pm 0.02 \mathrm{eV}$ and trap depth of $0.75 \pm 0.05 \mathrm{eV}$ are in excellent agreement with values measured from other unrelated experimental techniques.
\end{abstract}

Keywords: Deep level transient spectroscopy; Charge traps; Polymers; Poly(phenylene vinylene); Electroluminescence; Light emitting diodes

\section{Introduction}

Deep level transient spectroscopy (DLTS) is a traditional technique used to study traps in inorganic semiconductor devices which contain a depletion region (Schottky diodes, metal-insulator-semiconductor structures and heavily asymmetric junction diodes) $[1,2]$. The depletion region capacitance $C_{\mathrm{d}}$ can be directly related to the depletion region width $x_{\mathrm{d}}$ :

$x_{\mathrm{d}}=\frac{\varepsilon A}{C_{\mathrm{d}}}$

where $\varepsilon$ is the permittivity of the depleted material and $A$ is the device area. $x_{\mathrm{d}}$ depends upon the total amount of charge stored within the depletion region. This is not only the charge on the ionised dopant centres but also that in any bulk traps or interface states. $C_{\mathrm{d}}$ can therefore be used to measure the presence of traps. If any bulk traps or interface states intersect the quasi-Fermi level within the depletion region, the application of a forward bias voltage pulse will momentarily pull them below the Fermi level, causing them to be filled. DLTS uses the variation of the depletion region capacitance with time after such a voltage

\footnotetext{
${ }^{*}$ Corresponding author.

E-mail address: a.campbell@sheffield.ac.uk (A.J. Campbell).

${ }^{1}$ Current address: Institut für Angewandte Physik, Friedrich-SchillerUniversität Jena, Max-Wien-Platz 1, 07743 Jena, Germany.
}

pulse to measure the emptying of charge from any traps or interface states.

This technique obviously requires the presence of a depletion region. However, in nearly all polymer LEDs the density of accidental extrinsic impurity dopants is too low to form a depletion region type Schottky barrier smaller than the device thickness [3]. A clear exception arises in the ITO/poly ( $p$-phenylene vinylene) (PPV)/Al structures fabricated at the University of Bayreuth, Germany. In these devices the level of $\mathrm{InCl}_{3}$ p-type dopants is large enough to form a depletion region at the $\mathrm{PPV} / \mathrm{Al}$ interface [4]. Here, we report DLTS measurements of such $\mathrm{ITO} / \mathrm{PPV} / \mathrm{Al}$ devices.

\section{Experimental}

Devices were prepared as described in the literature [4]. Measurements were carried out using a Solartron Schlumberger SI1260 Impedance/Gain-Phase Analyser and an Oxford Instruments CCC1204 closed cycle, helium exchange gas cryostat. Results presented here are representative of typical devices.

\section{Results and discussion}

Fig. 1 shows the capacitance-voltage $(\mathrm{CV})$ characteristics of a $200-\mathrm{nm}$ thick device in the form of a $1 / C^{2}$ vs. 


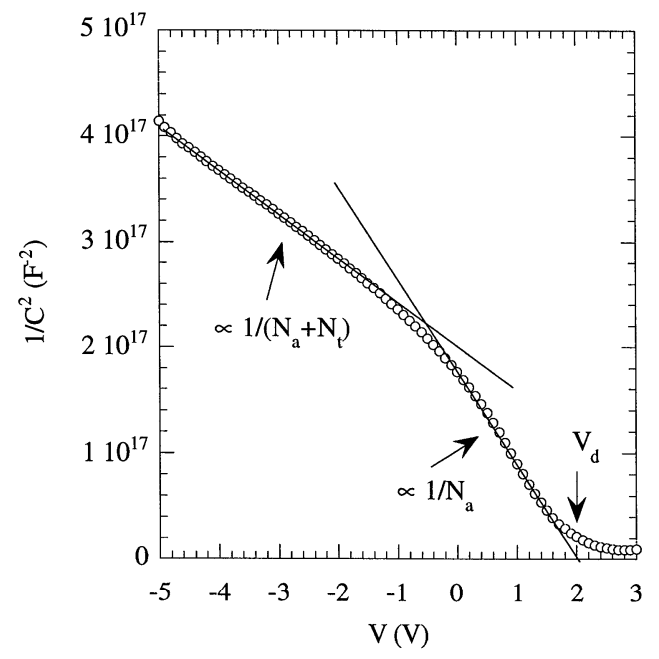

Fig. 1. Inverse capacitance squared, $1 / C^{2}$, against applied bias, $V_{\text {appl }}$, of an $\mathrm{ITO} / \mathrm{PPV} / \mathrm{Al}$ device.

$V$ plot. This behaviour is the classic characteristic for a Schottky diode that has majority carrier traps whose charged state, when empty, is of the same sign as the ionised dopant centres (see Fig. 6.17 in Ref. [2]). The larger gradient at positive bias arises from the $\mathrm{InCl}_{3}$ acceptor dopants, density $N_{\mathrm{a}}$, and is proportional to $1 / N_{\mathrm{a}}$, with an intercept that gives the diffusion potential $V_{\mathrm{d}}$. The smaller gradient at negative bias arises from the combination of acceptor dopants, $N_{\mathrm{a}}$, and those traps that can empty on the time scale of the experiment, $N_{t}\left(\omega_{\text {expt }}\right)$. This gradient is proportional to $1 /\left(N_{\mathrm{a}}+N_{\mathrm{t}}\left(\omega_{\text {expt }}\right)\right)$, where $\omega_{\text {expt }}$ is determined by the sweep rate of the measurement. The change in slope from $1 /\left(N_{\mathrm{a}}+N_{\mathrm{t}}\left(\omega_{\text {expt }}\right)\right)$ to $1 / N_{\mathrm{a}}$ is when, with increasing positive bias, the traps are pulled below the quasi-Fermi level at the interface, filling and no longer responding to the measurement. The results indicate that these devices contain bulk traps of the acceptor type (neutral when full, negatively charged when empty) with a density $N_{\mathrm{t}}>N_{\mathrm{a}}$. The measured values of the acceptor density, zero bias depletion width and diffusion potential for the devices in this work are very similar to those reported in the literature [4].

Fig. 2 shows the change of the capacitance with time on a $\log$-linear plot. This transient is very typical of all those observed. The length of the transient (the traps are still emptying after $7 \mathrm{~h}$ ) suggests that the traps are very deep within the energy gap. The sign of the transients shows that the amount of positive charge in the depletion region is decreasing with time, indicating that we are probing p-type majority carrier traps. The decay rate shows hardly any change as the quiescent bias level is varied, indicating that the transients are from bulk traps rather than interface states. All of the observed transients are also non-exponential, indicating that either the positive carrier transport states, the traps, or both, are distributed in energy.

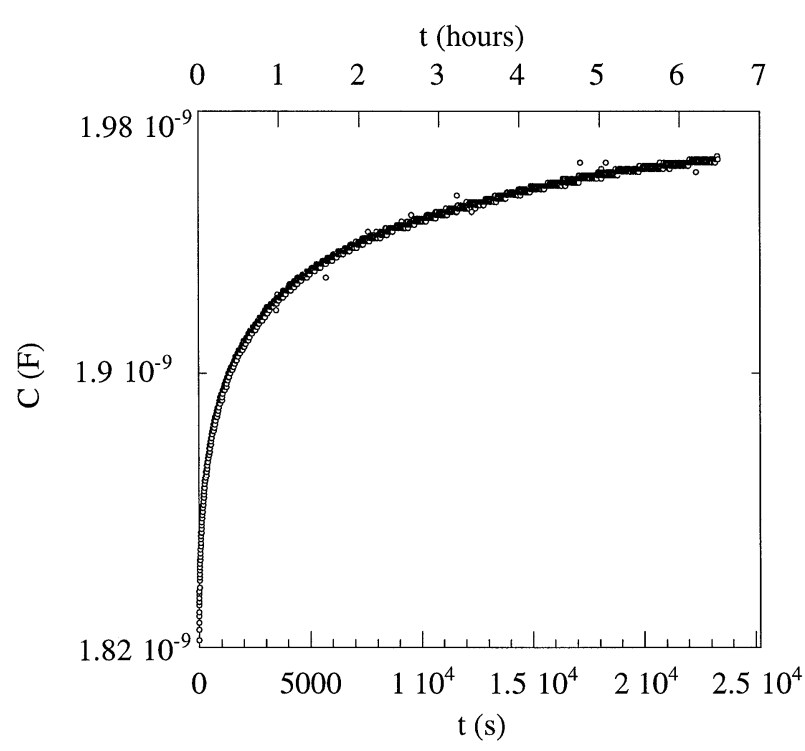

Fig. 2. Log-linear plot of transient capacitance, $C$, against time, $t$.

As shown by its optical absorption spectrum, PPV is a disordered molecular solid. Site-selective fluorescence measurements and an analysis of charge carrier mobility measurements suggest a characteristic Gaussian density of states (DOS) distribution with a standard deviation $\sigma$ of about 0.1 eve $[5,6]$. A similar transport state distribution has been suggested for other disordered organic materials [7]. The capacitance transients have, therefore, been modelled on the de-trapping of positive carriers from a discrete trap level to a Gaussian distribution of transport states as shown in Fig. 3.

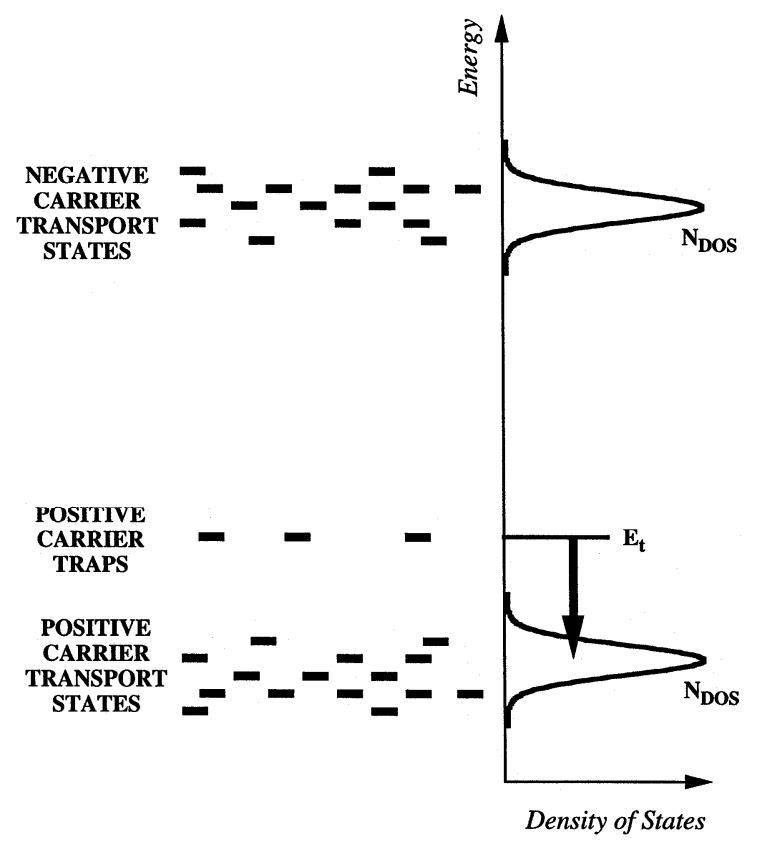

Fig. 3. Model of electronic structure of PPV used in this work to explain the transient capacitance. A discrete positive carrier trap level lies within the energy gap between Gaussian distributions of positive and negative carrier transport states. 


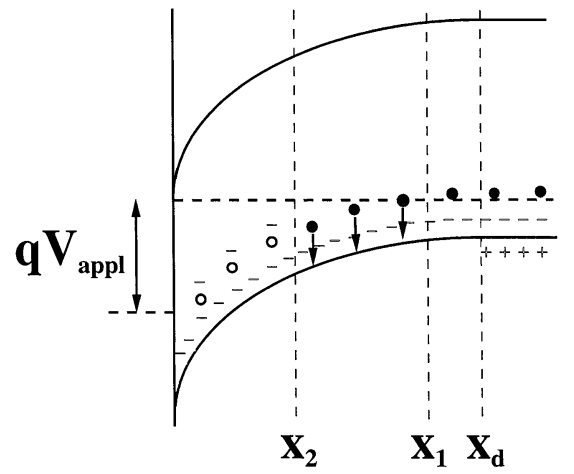

Fig. 4. Physical picture of the depletion region during trap emptying.

As $N_{\mathrm{t}}>N_{\mathrm{a}}$ the small trap density approximation normally used for DLTS cannot be used. The change of the depletion width $x_{\mathrm{d}}(t)$ with time is calculated from:

$$
\begin{aligned}
& \frac{2 \varepsilon_{\mathrm{r}} \varepsilon_{0}}{q}\left(V_{\mathrm{d}}-V_{\mathrm{appl}}+V^{*}\right) \\
& =N_{\mathrm{a}} x_{\mathrm{d}}(t)^{2}+N_{\mathrm{t}} x_{1}(t)^{2}-N_{\mathrm{t}} f(t)\left\{x_{1}(t)^{2}-x_{2}^{2}\right\}
\end{aligned}
$$

This model assumes the depletion region approximation. $V_{\text {appl }}$ is the applied quiescent bias. $V^{*}$ is a fitting parameter that allows for charge stored in fast interface states or fast bulk traps or for spatial variations in $N_{\mathrm{t}}$ (experimentally, this is dominated by the latter). $x_{1}(t)$ is the distance from the cathode to the point at which the trap level intersects the quasi-Fermi level when the device is held at quiescent bias (see Fig. 4). $x_{2}$ is the same distance during the filling voltage pulse. $f(t)$ is the variation with time of the trapped charge between $x_{1}(t)$ and $x_{2}$ [2]. $x_{1}(t)$ and $x_{2}$ are calculated from the depletion width by subtracting the parameter $\lambda$ where:

$\lambda=\sqrt{\frac{2 \varepsilon\left(E_{\mathrm{t}}-E_{\mathrm{F}}\right)}{q N_{\mathrm{a}}}}$

It also assumes that $N_{\mathrm{a}}$ does not vary with distance from the cathode as shown by the very linear $1 / C^{2}$ vs. $V$ plots of similar devices [4]. The p-type carrier mobility in the Bayreuth PPV indicates that the average transit time across the depletion region should be $\approx 10 \mu \mathrm{s}$ [8]. It is, therefore, assumed for simplicity that re-trapping is negligible on the time scales of the experiment. The decay function $f(t)$ is calculated by integrating at different times $t$ over a normalised Gaussian distribution $N_{\text {Gauss }}\left(g_{\mathrm{t}}\right)$ of trap depths $g_{\mathrm{t}}+E_{\mathrm{t}}$ :

$f(t)=\int N_{\text {Gaussian }}\left(g_{\mathrm{t}}\right) \exp \left\{-v \exp \left(\frac{-\left(g_{\mathrm{t}}+E_{\mathrm{t}}\right)}{k T}\right) t\right\} \mathrm{d} g_{\mathrm{t}}$

This corresponds to the physical picture in Fig. 3, with $v$ the attempt-to-escape frequency.

In inorganic semiconductors $v$ is calculated by assuming a model based on the capture of a ballistic particle moving in an energy band and has a temperature dependence of the form $T^{2} \exp \left(-E_{\text {capt }} / k T\right)$ [2]. Given that this is a disordered molecular material in which the trap capture cross-section is defined by the physical size of the trapping site, the density of states $N_{\text {DOs }}$ is the molecular density and conduction is by hopping transport; in this work, it is assumed that $v$ has a temperature dependence of the form $\exp \left(-E_{\mathrm{a}} / k T\right)$, where $E_{\mathrm{a}}$ is an activation energy associated with the zero-field carrier thermal velocity. $E_{\mathrm{a}}$ is expected to be about an order of magnitude less than $E_{\mathrm{t}}$ and should be contained within the estimated error in the latter of $\pm 0.05 \mathrm{eV}$.

$f(t)$ was calculated for a range of different $E_{\mathrm{t}}, v$ and Gaussian widths $\sigma$. Eq. 2 can be reduced to a quadratic form to find $x_{\mathrm{d}}(t)$. Eq. 1 can then be used to calculate the variation of $C_{\mathrm{d}}$ with $t$. A fit to the results is then obtained by varying $V^{*}$ and $N_{\mathrm{t}}$. The relative permittivity was taken to be $\varepsilon_{\mathrm{r}}=3.8$, as previously measured [4]. $N_{\text {Dos }}$ was taken as $8 \times 10^{20} \mathrm{~cm}^{-3}$ as previously calculated [3].

Fig. 5 shows the fit of our model to the transient shown in Fig. 2. An excellent match is obtained here as was also the case for all of the other measured transients. As shown in Fig. 5, the model constrains the Gaussian width $\sigma$ to be $0.10 \pm 0.02 \mathrm{eV}$, which is in excellent agreement with other estimates in the literature.

Fig. 6 shows capacitance transients recorded at 300, 280 and $260 \mathrm{~K}$ fitted to the model with a constant $N_{\mathrm{t}}$. The value of $N_{\mathrm{a}}$ used at each temperature was measured from the corresponding $C V$ plots. All of the results gave a trap depth $E_{\mathrm{t}}=0.75 \pm 0.05 \mathrm{eV}$ and an attempt-to-escape frequency $v=10^{8} \mathrm{~s}^{-1}$. The trap depth deduced is in very good agreement with the energies found from p-type carrier ToF activation and thermally stimulated current (TSC) measurements on the same Bayreuth PPV $[8,9]$.

Capacitance-voltage measurements of the devices in this work, recorded after exposure to air, showed that

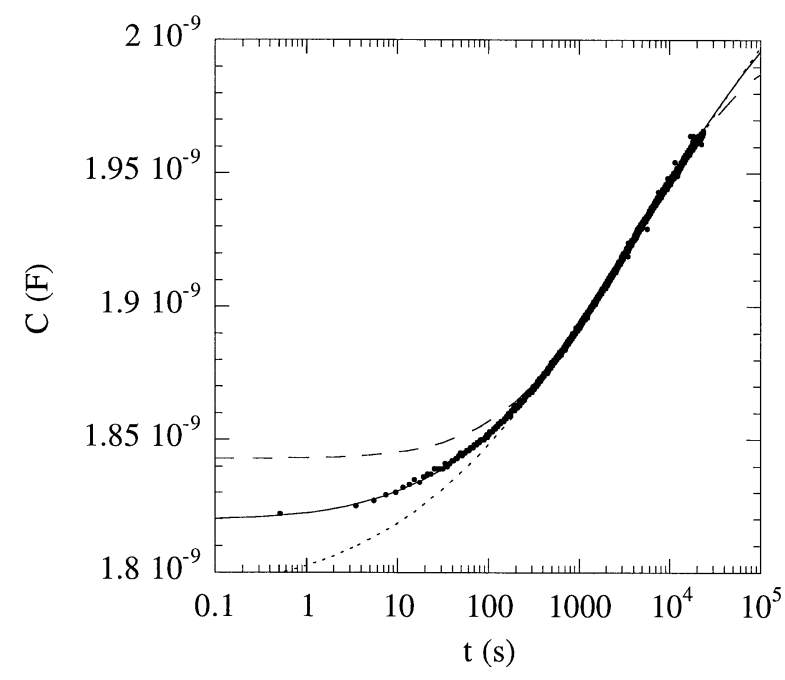

Fig. 5. Fit of model to results in Fig. 2 on a linear-log plot. Different fits are for a Gaussian DOS width, $\sigma$, of 0.06 (dashed line), 0.10 (full line) and $0.14 \mathrm{eV}$ (dotted line). 


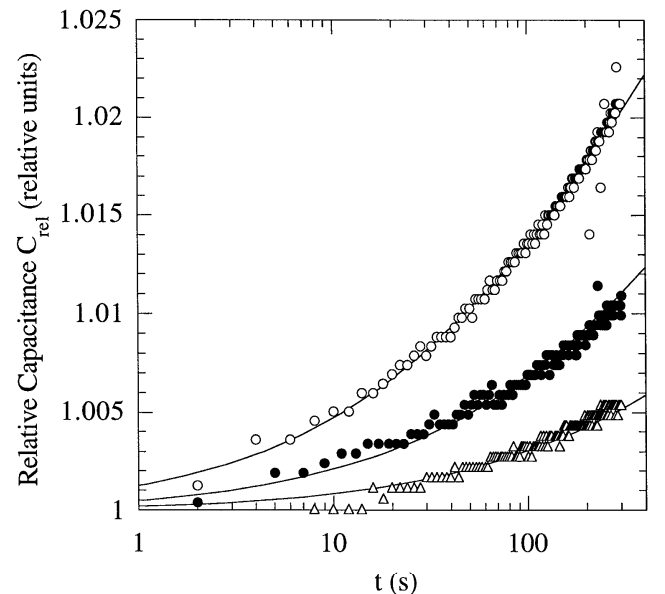

Fig. 6. Variation of relative capacitance, $C_{\text {rel }}$, against time, $t$, at 300 (open circles), 280 (filled circles) and $260 \mathrm{~K}$ (open triangles). Solid lines are fits to model at these temperatures. Data and fits have been normalised to $C$ of fits at $t=10^{-5} \mathrm{~s}$ for illustration purposes.

under He atmosphere $N_{\mathrm{t}}$ decreases with time over a number of days, implying the traps are due to the presence of water and/or oxygen. This is in good agreement with the TSC measurements [9]. Oxygen is generally considered to be an n-type carrier trap in molecular materials, so water is strongly implicated. This is supported by a joint spectroscopic and theoretical study of PPV [10].

At a distance between 30 and $50 \mathrm{~nm}$ from the cathode (modelled for $E_{\mathrm{t}}=0.75 \mathrm{eV}$ ) $N_{\mathrm{t}}$ is found to be of order 4 to $7 \times 10^{17} \mathrm{~cm}^{-3}$. The spatial variation of $N_{\mathrm{t}}$ was investigated by using different quiescent bias levels with a constant pulse height. The results showed a constant increase in $N_{\mathrm{t}}$ with distance from the cathode.

\section{Conclusion}

DLTS and $C V$ measurements on PPV light emitting Schottky diodes show the presence of bulk majority positive carrier traps of the acceptor type (neutral when full, negatively charged when empty). The capacitance tran- sients have been successfully modelled on the decay of a discrete trap state, with depth $0.75 \pm 0.05 \mathrm{eV}$, to a Gaussian distribution of transport states with standard deviation $0.10 \pm 0.02 \mathrm{eV}$. This is in very good agreement with results in the literature from other unrelated experimental techniques. The traps are related to the presence of water and/or oxygen, and have a high density $\approx 5 \times 10^{17} \mathrm{~cm}^{-3}$ that increases with increasing distance from the cathode.

\section{Acknowledgements}

We gratefully acknowledge funding from the UK Engineering and Physical Sciences Research Council (GR/K57428) and from Dow Chemical for support of the work in Sheffield. We also thank J. Gmeiner for synthesis of the PPV precursor and the Bayerische Forschungsstiftung (FOROPTO) and the Sonderforschungsbereich 481 for funding the work at Bayreuth. A British Council/ DAAD Academic Research Collaboration grant funded the reciprocal exchange visits between Sheffield and Bayreuth.

\section{References}

[1] D.V. Lang, J. Appl. Phys. 45 (1974) 3023.

[2] P. Blood, J.W. Orton, The Electrical Characterisation of Semiconductors: Majority Carriers and Electron States, Academic Press, London, 1992.

[3] A.J. Campbell, D.D.C. Bradley, D.G. Lidzey, J. Appl. Phys. 82 (1997) 6326

[4] M. Meier, S. Karg, W. Reiss, J. Appl. Phys. 82 (1997) 1961.

[5] U. Rauscher, H. Bässler, D.D.C. Bradley, M. Hennecke, Phys. Rev. B 42 (1990) 9830.

[6] E.M. Conwell, Y.N. Gartstein, SPIE 2528 (1995) 23

[7] P.M. Borsenberger, D.S. Weiss, Organic Photoreceptors for Xerography, Marcel Dekker, New York, 1998.

[8] E. Lebedev, Th. Dittrich, V. Petrova-Koch, S. Karg, W. Brütting, Appl. Phys. Lett. 71 (1997) 2686.

[9] M. Meier, S. Karg, K. Zuleeg, W. Brütting, M. Schwoerer, J. Appl. Phys. 84 (1998) 87.

[10] K. Xing, M. Fahlman, M. Lögdlund, D.A. dos Santos, V. Parenté, R. Lazzaroni, J.L. Brédas, R.W. Gymer, W.R. Salaneck, Adv. Mater. 8 (1996) 971. 